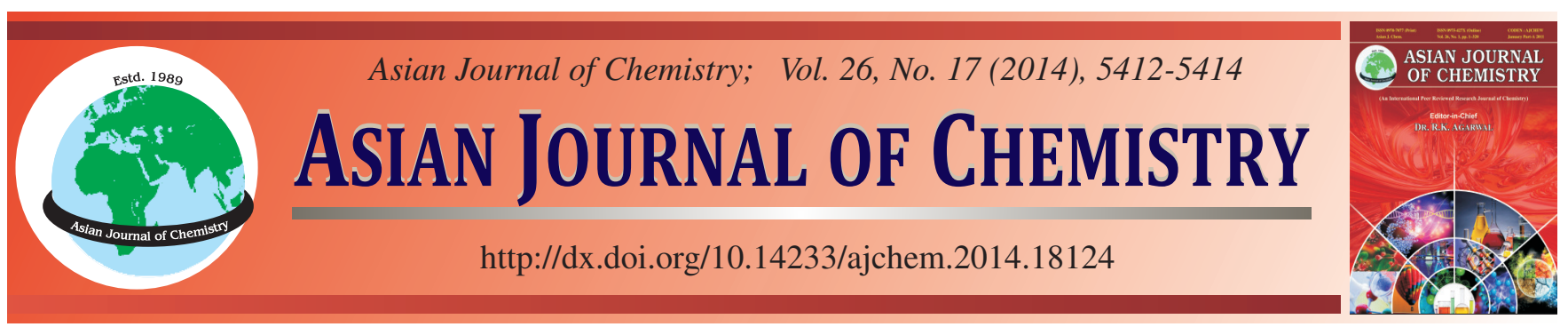

\title{
Hydrophobic Modified Nanosized Crosslinked Polymer Microspheres $\dagger$
}

\author{
Hailing Chen ${ }^{1, *}$, XiaOyu ZhenG ${ }^{2}$, Qinzhe JiAng ${ }^{2}$ and XiAnJie $\mathrm{Li}^{3}$
}

${ }^{1}$ School of Science, China University of Petroleum (Beijing), Beijing, China; Department of Biological and Chemical Engineering, Nanyang Institute of Technology, Nanyang, P.R. China

${ }^{2}$ School of Science, China University of Petroleum (Beijing), P.R. China

${ }^{3}$ State Key Laboratory of Offshore Oil Exploitation, CNOOC Research Institute, Beijing, P.R. China

*Corresponding author: Tel./Fax: +86 1089733782 E-mail: hailiingchen2@gmail.com

\begin{abstract}
The properties of nanoparticles are not only dependent on their size and shape, but also are determined by surface properties. To increase shearing resistance, hydrophobic monomer is introduced to inverse microemulsion polymerization and hydrophobic-modified crosslinked polymer nanometer microspheres $\left(\mathrm{Q}_{18}\right)$ were obtained. As the object of the comparative study, non-hydrophobic cross-linked polymer microspheres $\left(\mathrm{Q}_{5}\right)$ were also made. The microscopic morphology, size distribution and plugging effect of $\mathrm{Q}_{18}$ and $\mathrm{Q}_{5}$ were studied. The experimental results showed that, the morphology of $\mathrm{Q}_{18}$ and $\mathrm{Q}_{5}$ are spherical with polydispersion, the hydrophobic associations come into being in aqueous solution above a particular concentration, so the particle size distribution model of $\mathrm{Q}_{18}$ deviates from double logarithmic distribution model which matches to non-hydrophobic cross-linked polymer microspheres $\left(\mathrm{Q}_{5}\right)$. $\mathrm{Q}_{18}$ can enter deeper in the sand-filled pipe than $\mathrm{Q}_{5}$ to form plugging.
\end{abstract}

Keywords: Cross-linked polymer microspheres, Microstructure, Hydrophobic modification, Particle size distribution, Plugging effect.

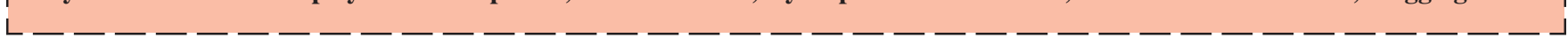

\section{INTRODUCTION}

Polymer nanomaterials with sizes in the range of 1-1000 $\mathrm{nm}$ are at least one dimension, have attracted much attention because of their dramatically increased surface area to volume ratio, leading to a variety of different properties ${ }^{1,2}$. The nanosized structures afford polymers unique and supreme properties differing from those of the bulk polymer, such as optical, magnetic, thermal and electrical properties. Polymer nanomaterials are potentially used in drug delivery, surface coatings, nanoreactors, catalysis and filtration. Many scholars believe that nanomaterials will be one of the most promising materials in the 21 st century, especially the polymer nanomaterials. In recent years nanometer materials have been widely used for drilling fluid, the profile control, oil displacement and wastewater treatment in the oil and gas fields ${ }^{3}$. Cross-linked polymer microspheres, whose crosslinking degree is controllable, can enter the deep of the oil reservoir to carry on the profile, can effectively increase the swept volume of injected water and can adjust injection profile to reduce heterogeneity, so as to increase oil recovery significantly ${ }^{4,5}$. The size and size distribution play a vital role in plugging process, as well as viscoelasticity of microspheres ${ }^{6}$. Great breakthrough in the flowing performances of hydrophobically modified polyamide has been obtained ${ }^{7-9}$. Here a hydrophobic chain was presented to cross-linked polymer microspheres to observe the association in aqueous solution and plugging performance.

\section{EXPERIMENTAL}

Acrylamide (AM), sodium acrylate (SAA), 2-acrylamido2-methylpropane sulfonate (AMPS), acryloyloxyethyltrimethyl ammonium chloride (DAC), white oil (260\# solvent oil), polyethylene glycol sorbitan monostearate (Tween-60) and sorbitan (Z)-mono-9-octadecenoate (Span-80) were commercially available industrial products. Crosslinking monomer (CM), with two double bonds on both ends of the molecule chain, contains groups of alkyl and polyoxyethylene, hydrophobic monomer (HM) is octadecyl fatty alcohol polyoxyethylene ether and the both were lab homemade. Deionized water was used throughout. Other reagents were analytically pure and were used without further purification.

General procedure: The hydrophobic crosslinked polymer microsphere $\left(\mathrm{Q}_{18}\right)$ was prepared by inverse microemulsion polymerization ${ }^{10,11}$. Water phase solution was prepared by mixing AM (12.939 g), SAA (3.664 g), AMPS (16.524 g) and $\mathrm{CM}(0.690 \mathrm{~g})$ in water $(16.524 \mathrm{~g}), \mathrm{NaOH}$ and acetic acid were 
used to control the solution $\mathrm{pH}$ between 8.1 and 8.2. HM (1.545 g), Span-80 (13.6 g) and Tween-60 (8.9 g) were dissolved in white oil (27.5 g) and then oil phase solution was placed in a reaction kettle, which was equipped with a mechanical stirrer, a nitrogen inlet and an outlet. Aqueous solution was then added to the kettle slowly, the mixture was stirred for $1 \mathrm{~h}$ at $7^{\circ} \mathrm{C}$. The electrical conductivity was measured to ensure that the solution was water-in-oil microemulsion (usually $<0.5 \mu \mathrm{S} / \mathrm{cm}$ ). The kettle was purged with $\mathrm{N}_{2}$ for another $1 \mathrm{~h}$. The mixture was heated to $23{ }^{\circ} \mathrm{C}$ under a nitrogen atmosphere, $0.375 \mathrm{~g}$ of 0.1 wt $\% \mathrm{NH}_{4} \mathrm{~S}_{2} \mathrm{O}_{8}$ solution was added and a small amount of $\mathrm{SO}_{2}$ gas was then brought in. The polymerization proceeded for $4 \mathrm{~h}$ at $60^{\circ} \mathrm{C}$. Four volumes of ethanol were added with stirring to precipitate the polymers after the reaction mixture was cooled down. The polymers were washed with acetone twice and then extracted with n-heptane using a soxhlet extractor for two days, in order to remove all traces of water, surfactant, residual monomers and initiator. Finally, the polymers were dried under vacuum at $60{ }^{\circ} \mathrm{C}$ for three days.

With DAC substituted for the hydrophobic monomer HM, $\mathrm{Q}_{5}$ was synthesized and the reaction conditions and purification method were the same as those described above.

Detection method: The sizes and distributions of polymer microspheres and associating aggregates, hydrophobic associations and plugging performance were investigated with Quanta 200F environment scan electron microscope (FEI, America), laser particle analyzer (SALD2300, Japan), fluorescence spectroscopy (RF-5001PC, Japan) and sand-filled tube physical model ${ }^{4}$.

\section{RESULTS AND DISCUSSION}

Fig. 1 shows images obtained through environment scan electron microscope (ESEM). The morphology of polymer microspheres dissolved in water is spherical.

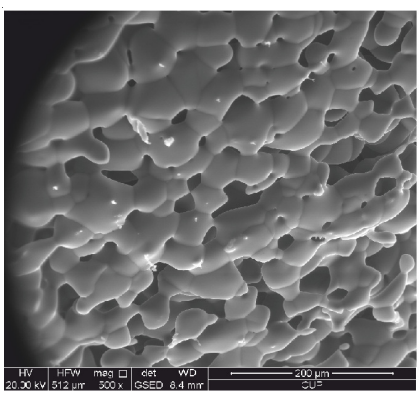

(a) Q5

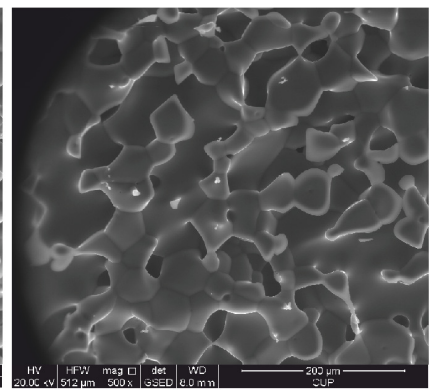

(b) Q18
Fig. 1. ESEM photos of polymer microspheres

Interestingly, as shown in Fig. 2, the size distributions of $\mathrm{Q}_{5}$ and $\mathrm{Q}_{18}$ are different, unimodal distribution for $\mathrm{Q}_{5}$, but bimodal for $\mathrm{Q}_{18}$. The sizes of the two polymer spheres all increase for about 10 times or more, which shows that the microspheres have good swelling performance in the water. After fitting both curves of size distributions by lognormal function, the fitting correlation coefficients and average particle sizes of the two are not same, for $\mathrm{Q}_{5}, \mathrm{R}^{2}=0.9734$, the average particle size $\mathrm{xc}$ $=1.49 \mu \mathrm{m}$. And for $\mathrm{Q}_{18}, \mathrm{R}^{2}=0.8543$, the average particle size $\mathrm{xc}=16.29 \mu \mathrm{m}$. All those show that due to the hydrophobic association, hydrophobic cross-link polymer microspheres,

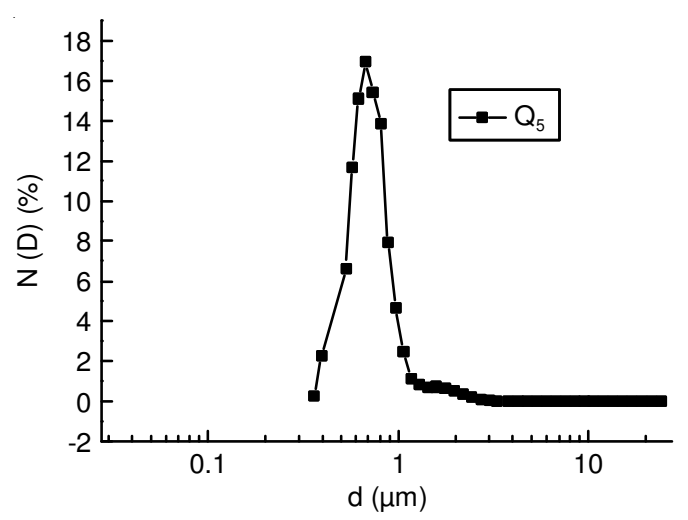

(a) $Q_{5}$

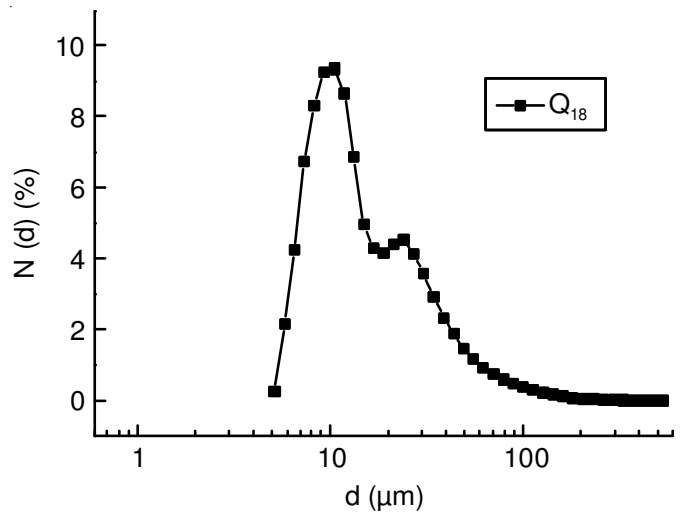

(b) $Q_{18}$

Fig. 2. Size distribution of polymer microspheres after swelled in water for 5 days

$\mathrm{Q}_{18}$, cooperate into larger aggregate particles and the distribution deviates from the logarithmic normal distribution more than that of non-hydrophobic cross-linked polymer microspheres $\left(\mathrm{Q}_{5}\right)$.

Fluorescence spectrophotometer is the most powerful tool to study of intermolecular hydrophobic association ${ }^{12,13}$. In the fluorescence spectra of pyrene molecular used as the probe, the intensity ratio of the first one and the third peak, $I_{1} / I_{3}$, is sensitive to the polarity variation of environment. As the value of $\mathrm{I}_{1} / \mathrm{I}_{3}$ decreases, polarity condition of pyrene also decreases. Prepare the following aqueous solution, which contain the mass of the polymer microspheres are $0,100,500,1500,2000,2500$, $3000,3500,4000 \mathrm{mg} \mathrm{L}^{-1}$, respectively. Among them, the concentration of pyrene is $5 \times 10^{-6} \mathrm{~mol} \mathrm{~L}^{-1}$. With the increase of the polymer microspheres' concentration, the value of $\mathrm{I}_{1} / \mathrm{I}_{3}$ gradually decreases (Fig. 3), that shows hydrophobic microzone is gradually formed in the solution. After the mass fraction is more than $0.15 \%$, the values of $I_{1} / I_{3}$ level off, the result shows uniform hydrophobic microzones have created in the solution. In addition, when the mass fraction is over $0.3 \%$, the value of $\mathrm{I}_{1} / \mathrm{I}_{3}$ quickly drops, as indicates that large hydrophobic micro zones are destroyed, but net structure forms in the solution.

The mass fraction of $400 \mathrm{mg} \mathrm{L}^{-1}$ cross-linked polymer microspheres dispersed system prepared with $0.8 \% \mathrm{NaCl}$ solution were swelled for 10 days under $60^{\circ} \mathrm{C}$ and then injected into the sand-filled tube. Fig. 4 showed the pressures of different positions along with sand-filled tube changed with the injection volume of microsphere dispersion system. Among 
TABLE-1

PLUGGING EXPERIMENTAL PARAMETERS OF SAND-FILLED TUBE

\begin{tabular}{cccccc}
\hline \multirow{2}{*}{ Disperse system } & $\begin{array}{c}\text { Concentration of } \\
\text { microspheres }\left(\mathrm{mg}^{-1}\right)\end{array}$ & $\begin{array}{c}\text { Permeability of sand- } \\
\text { filled tube }\left(10^{-3} \mu \mathrm{m}^{2}\right)\end{array}$ & $\begin{array}{c}\text { Plugging } \\
\text { position }\end{array}$ & $\begin{array}{c}\text { Resistance } \\
\text { coefficient }\end{array}$ & $\begin{array}{c}\text { Residual resistance } \\
\text { factor }\end{array}$ \\
\hline $\mathrm{Q}_{5}$ & 400 & 1241 & middle & 5.58 & 4.52 \\
$\mathrm{Q}_{18}$ & 400 & 1063 & back & 9.60 & 6.40 \\
\hline
\end{tabular}
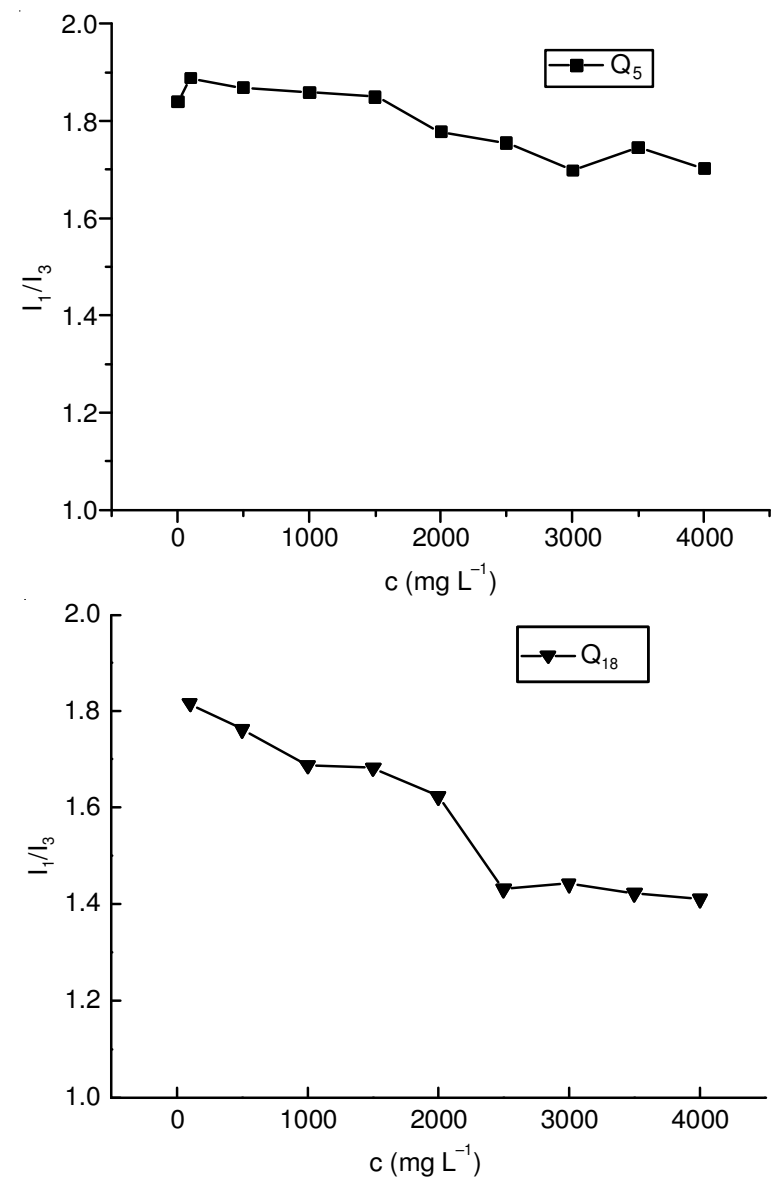

Fig. 3. $I_{1} / I_{3}$ values of pyrene changing with the mass fraction of hydrophobic microspheres

them, the P1 was pressure differential for the front $20 \mathrm{~cm}$ of sand-filled tube, the P2 for the middle $20 \mathrm{~cm}$ and the P3 for the back $20 \mathrm{~cm}$. Fig. 4 and Table-1 showed that hydrophobic cross-linked microspheres $\left(\mathrm{Q}_{18}\right)$ can enter more deeply than non-hydrophobic cross-linked microspheres $\left(\mathrm{Q}_{5}\right)$ and has higher resistance coefficient and residual resistance facto, so hydrophobic cross-linked microspheres have more perfect plugging performance than non-hydrophobic cross-linked microspheres.

\section{Conclusion}

The morphology of $\mathrm{Q}_{18}$ and $\mathrm{Q}_{5}$ are all spherical with a polydisperse distribution of particle size. Hydrophobic association can appear when $\mathrm{Q}_{18}$ dissolves in water, so particle size distribution deviates more from the logarithmic normal distribution, which matches $\mathrm{Q}_{5}$ well. It is also proved by fluorescence spectrophotometer that $\mathrm{Q}_{18}$ could associated in aqueous, but $\mathrm{Q}_{5}$ could not. $\mathrm{Q}_{18}$ can enter more deeply than $\mathrm{Q}_{5}$ and has higher resistance coefficient and residual resistance factor.
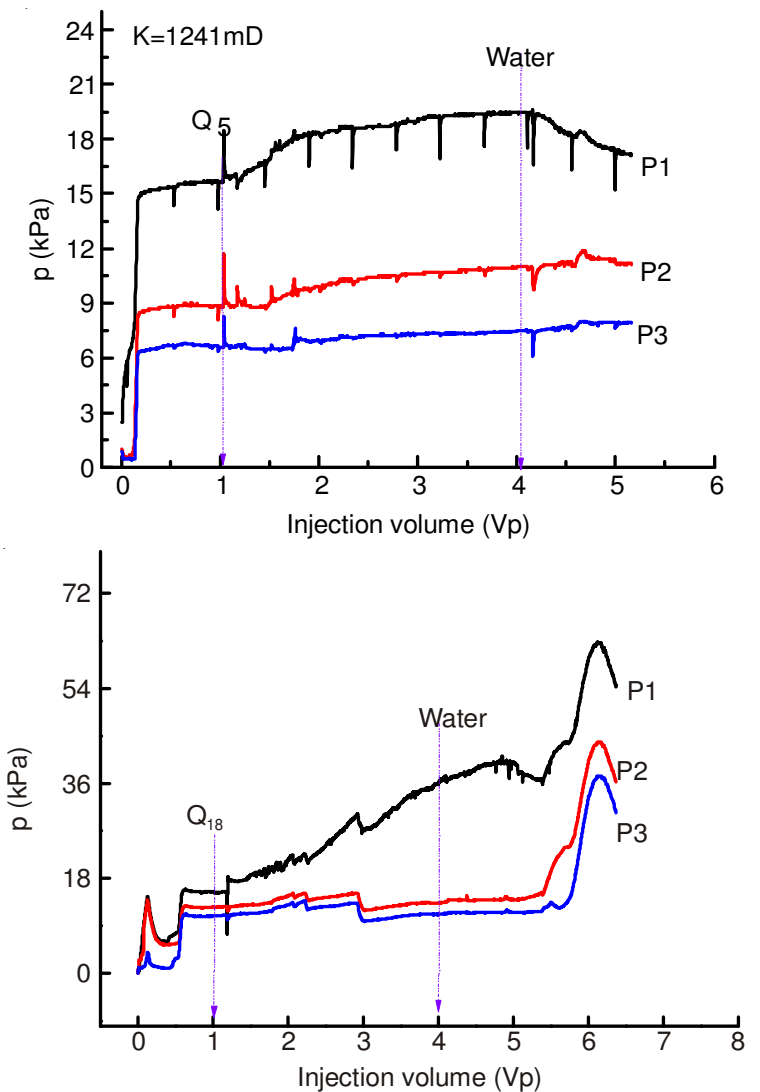

Fig. 4. Flow pressure differential curves of cross-linked polymer microsphere dispersions. $* \mathrm{Vp}$ is the total volume of all pores in sand-filled tube

\section{ACKNOWLEDGEMENTS}

This work was supported by the National Science and Technology Major Project (Grant No. 2011ZX05024-004-05).

\section{REFERENCES}

1. R. Fu and G.-D. Fu, Polym. Chem., 2, 465 (2011).

2. C.L. Bai and M.H. Liu, Angew. Chem. Int. Ed., 52, 2742 (2013).

3. Z. Peng, Z.H. Wang, H.J. He and J.D. Li, Adv. Fine Petrochem., 12, 8 (2011).

4. X.Y. Zheng, J. Zhang, J. Sun, H. Ming, L. Song and J.R. Yang, Oilfield Chem., 29, 172 (2012).

5. H.L. Chen, X.Y. Zheng and Y. Wang, Modern Chem. Ind., 31, 62 (2011).

6. G.L. Lei, W.Z. Li, X.F. Jia, C.J. Yao and W.K. Sun, Petroleum Geology Recovery Efficiency, 19, 41 (2012).

7. E. Volpert, J. Selb and F. Candau, Polymer (Guildf.), 39, 1025 (1998).

8. Y. Lin, L. Kaifu and H. Ronghua, Eur. Polym. J., 36, 1711 (2000).

9. C. Zhong, P. Luo and L. Jiang, J. Solution Chem., 39, 355 (2010).

10. F. Candau, Z. Zekhnini and F. Heatley, Macromolecules, 19, 1895 (1986).

11. J. Barton, J. Prog. Polym. Sci., 21, 399 (1996).

12. F.M. Winnik, Chem. Rev., 93, 587 (1993).

13. C.R. Zhong, P.Y. Luo and X.F. Lian, J. Solution Chem., 42, 1863 (2013). 